\title{
ON ANALYTIC CONTINUABILITY \\ OF THE MISSING CAUCHY DATUM FOR HELMHOLTZ BOUNDARY PROBLEMS
}

\author{
MIRZA KARAMEHMEDOVIĆ \\ (Communicated by Richard Rochberg)
}

\begin{abstract}
We relate the domains of analytic continuation of Dirichlet and Neumann boundary data for Helmholtz problems in two or more independent variables. The domains are related à priori, locally and explicitly in terms of complex polyrectangular neighbourhoods of planar pieces of the boundary. To this end we identify and characterise a special subspace of the standard pseudodifferential operators with real-analytic symbols. The result is applicable in the estimation of the domain of analytic continuation of solutions across planar pieces of the boundary.
\end{abstract}

\section{INTRODUCTION}

For a second-order elliptic boundary problem in two or more independent variables, with only the Dirichlet or the Neumann condition specified, to what subset of the complexified boundary can the missing Cauchy datum be continued analytically? In view of existence results such as the theorem of Cauchy-Kovalevsky [5, Theorem 9.4.5] or the propagation of singularities of solutions to the analytic Cauchy problem [24, this question is central in the à priori estimation of the domain of analytic continuability of solutions across the boundary. This, in turn, is applicable, e.g., in the stability and convergence analysis of 'interior source methods', which is, a family of promising numerical methods for direct and inverse elliptic boundary problems $[2,3,8,11$.

In Millar [14 22, boundary integral representations of the solution are used to relate the Cauchy data, and the domain of analytic continuability of the missing boundary datum is estimated globally, essentially by analytic continuation of the integrals to complex space. This is done for general linear, elliptic, second-order, analytic, exterior boundary problems in two independent variables and with piecewise analytic boundary in [18, for such interior and exterior problems with analytic boundary in [21], as well as for exterior three-dimensional Helmholtz problems in a half-space [19] or with axisymmetric boundary [20]. Another global approach can be found in Section 4 of Sternin and Shatalov [25, for three-dimensional Helmholtz problems with Neumann datum given on an algebraic surface; see Sternin and Shatalov [24] for a more general treatment. More recently, Kangro, Kangro and Nicolaides [23] proposed a local approach to the à priori analytic continuation of solutions of two-dimensional Dirichlet boundary problems for the Helmholtz equation across analytic pieces of the boundary. Implicit in the method (see the comment

Received by the editors May 19, 2010 and, in revised form, November 17, 2012.

2010 Mathematics Subject Classification. Primary 35S05. 
immediately after Lemma 1 on p. 594 of [23]) is a regularity result regarding the missing Neumann datum, given essentially as follows: if both a parametrisation of the considered piece of the boundary and the (localised) Dirichlet datum can be analytically continued to a complex rectangle $\{t+i \eta \in \mathbb{C}, t \in[a, b], \eta \in[-c, c]\}$, then so can the missing (localised) Neumann datum. Kangro and Kangro [7] suggest a method analogous to that of 23 to handle locally the analytic continuation of solutions to three-dimensional Dirichlet problems for the Helmholtz equation across planar pieces of the boundary. There, they propose that if the Dirichlet datum can be analytically continued to the complex polyrectangle

$$
\left\{\left(x+i \xi_{1}, y+i \xi_{2}\right), x \in\left[a_{1}-2 r, a_{1}+2 r\right], y \in\left[a_{2}-2 r, a_{2}+2 r\right], \xi_{1}, \xi_{2} \in[-r, r]\right\}
$$

in $\mathbb{C}^{2}$, then the missing Neumann datum is analytically continuable to the set

$$
\left\{\left(x+i \xi_{1}, y+i \xi_{2}\right), x \in\left[a_{1}-r, a_{1}+r\right], y \in\left[a_{2}-r, a_{2}+r\right], \xi_{1}^{2}+\xi_{2}^{2} \leq r^{2}\right\}
$$

The method of [7, 23] is, in principle, extendible to higher dimensions. However, as stated on p. 592 of [23], '[...] in more than two dimensions the computations become quite involved.'

We here estimate the domain of analytic continuation of the missing Cauchy datum on open planar subsets of the boundary for Dirichlet and Neumann problems for the Helmholtz equation in $n+1$ independent variables, for any $n \in \mathbb{N}$. The analysis is à priori (that is, it does not require a solution of the boundary problem) and local, and in particular it uses no information about the boundary or about the Cauchy data outside the planar subset. To prove the main result, we identify and characterise a special subspace of the standard pseudodifferential operators on $\mathbb{R}^{n} \times \mathbb{R}^{n}$. The standard pseudodifferential operators are described, e.g., in Chapter XVIII of Hörmander [6]. The remainder of this section contains some notational conventions, a precise statement of the main result, and an overview of the rest of the paper.

In the following, $n$ is a fixed positive integer. A function $f$ defined on an open subset $\Omega$ of $\mathbb{R}^{n}$, or of $\mathbb{C}^{n}$, is here said to be real-analytic in $\Omega$, respectively analytic in $\Omega$, if for every point $x$ in $\Omega$ there is a nonempty neighbourhood of $x$ (in $\mathbb{R}^{n}$ or $\mathbb{C}^{n}$, respectively, in which the Taylor series of $f$ about $x$ is convergent and agrees with $f$. Elements $z$ of $\mathbb{C}^{n}$ are written $\left(z_{1}, \ldots, z_{n}\right)$, and we adopt the notation $z_{(j)}=\left(z_{1}, \ldots, z_{j-1}, z_{j+1}, \ldots, z_{n}\right)$. Multiindices are understood to be $n$-tuples of nonnegative integers. For every multiindex $\alpha$, the convention is that $|\alpha|=\sum \alpha_{j}$. The operator $\Delta$ is the Laplacian on $\mathbb{R}^{n+1}, \Delta=\sum_{j=1}^{n+1} \partial_{j}^{2}$, and $\Delta+k^{2}$ is the Helmholtz operator on $\mathbb{R}^{n+1}$. The constant $k$ is assumed positive. Finally, $\mathscr{L}(A, B)$ is the vector space of continuous linear maps from $A$ to $B$.

Now fix $n$-tuples $\left.\left.a, b^{-}, b^{+} \in\right] 0, \infty\right]^{n}$, write $\left.\tau(a)=\prod_{j=1}^{n}\right]-a_{j}, a_{j}[$, and define the open complex polyrectangle

$$
T\left(a, b^{ \pm}\right)=\left\{z \in \mathbb{C}^{n}, \operatorname{Re} z \in \tau(a), \operatorname{Im} z \in \prod_{j=1}^{n}\right]-b_{j}^{-}, b_{j}^{+}[\}
$$


Fix $l \in\{0,1\}$ and $\lambda>0$, and assume $u \in C^{1}(\overline{\tau(a)} \times[0, \lambda])$ satisfies

$$
\left.\left(\Delta+k^{2}\right) u=0 \quad \text { in } \tau(a) \times\right] 0, \lambda[
$$

$\lim _{x_{j} \rightarrow \pm a_{j}} \partial_{j}^{r} u(\cdot, 0)$ analytically continuable to $T\left(a_{(j)}, b_{(j)}^{ \pm}\right)$and in $C^{1}\left(\overline{T\left(a_{(j)}, b_{(j)}^{ \pm}\right)}\right)$ for $r=0,1, j=1, \ldots, n$;

$\lim _{x_{n+1} \nearrow \lambda} \partial_{n+1}^{r} u$ analytically continuable to $T\left(a, b^{ \pm}\right)$and in $C^{1}\left(\overline{T\left(a, b^{ \pm}\right)}\right), r=0,1$;

$U_{l}:=\lim _{x_{n+1} \searrow 0} \partial_{n+1}^{l} u$ analytically continuable to $T\left(a, b^{ \pm}\right)$and in $C^{1}\left(\overline{T\left(a, b^{ \pm}\right)}\right)$.

The $C^{1}$ regularity above is meant as one-sided, from the interior of the domain, and in the case of traces of the solution $u$, it is understood w.r.t. the variables $\operatorname{Re} z_{j}$, $\operatorname{Im} z_{j}$. The function $U_{l}$ is the given boundary datum on $\tau(a)$. The main result here is the following.

Theorem 1.1. The missing boundary datum $U_{1-l}=\lim _{x_{n+1} \searrow 0} \partial_{n+1}^{1-l} u$ is analytically continuable to each complex polyrectangle $T\left(\widetilde{a}, \widetilde{b}^{ \pm}\right)$with

$$
\left.\widetilde{a}_{j} \in\right] 0, a_{j}\left[\quad \text { and } \tilde{b}_{j}^{ \pm} \in\right] 0, \min \left\{a_{j}-\widetilde{a}_{j}, b_{j}^{ \pm}\right\}[, \quad j=1, \ldots, n .
$$

The Helmholtz operator is invariant under translation and rotation in $\mathbb{R}^{n+1}$. Therefore, the setup of Theorem 1.1 can, without loss of generality, be understood as the localisation of a Helmholtz boundary problem about a point of the boundary where the latter is a subset of a hyperplane. The functions $U_{0}$ and $U_{1}$ are localisations of the corresponding Dirichlet and Neumann boundary datum, respectively.

The rest of this paper is organised as follows. In Section 2, we show a mapping property of a subspace of the standard pseudodifferential operators that are specially suited for a proof of Theorem 1.1. That section is inspired in part by the analysis of Boutet de Monvel [1. Section 3 contains a proof of Theorem 1.1 and involves the development on pp. 109-110 of Section 18.2 and pp. 232-236 of Section 20.1 in Hörmander [6]. Finally, in Section 4, we comment on the generalisation of Theorem 1.1

\section{A MAPPING PROPERTY OF}

CERTAIN STANDARD PSEUDODIFFERENTIAL OPERATORS

Throughout this section, $m$ is a real.

2.1. The space $S_{\mathrm{r}-\mathrm{a}}^{m, \varepsilon}$ of real-analytic symbols. The pseudodifferential symbols used in the proof of Theorem 1.1 are functions of only the cotangent variables in $T^{*} \mathbb{R}^{n}$. Recall that a function $p$ is in the standard symbol space $S^{m}\left(\mathbb{R}^{n}\right)$ if and only if $p \in C^{\infty}\left(\mathbb{R}^{n}\right)$ and for each multiindex $\alpha \in \mathbb{N}_{0}^{n}$ there is a constant $C_{\alpha}$ satisfying

$$
\left|\partial^{\alpha} p(\xi)\right| \leq C_{\alpha}(1+|\xi|)^{m-|\alpha|}, \quad \xi \in \mathbb{R}^{n} .
$$

In the following, we write $S^{m}$ for $S^{m}\left(\mathbb{R}^{n}\right)$. Also, $S^{-\infty}=\bigcap_{\mu \in \mathbb{R}} S^{\mu}$. With $\alpha \in \mathbb{N}_{0}^{n}$, the map taking each $p \in S^{m}$ to the smallest number $C_{\alpha}$ that satisfies (2.1) is a seminorm on $S^{m}$; write $\|\cdot\|_{\alpha}^{(m)}$ for this seminorm. The space $S^{m}$ is now equipped with the corresponding 'natural topology' $\mathfrak{T}^{m}$ : the coarsest topology on $S^{m}$ in which all the seminorms $\|\cdot\|_{\alpha}^{(m)}, \alpha \in \mathbb{N}_{0}^{n}$, are continuous, and in which the addition is continuous. Chapter XVIII of Hörmander [6] contains a treatment of the standard pseudodifferential symbols and operators. 
Fix $R \geq 0$ and $\varepsilon \in] 0,1[$, and let

$$
K_{\varepsilon}=\{\zeta \in \mathbb{C},|\operatorname{Im} \zeta|<\varepsilon|\operatorname{Re} \zeta| \text { if }|\operatorname{Re} \zeta|>R, \operatorname{Im} \zeta=0 \text { otherwise }\} .
$$

That a function $p$ is 'analytic on $K_{\varepsilon}^{n}$ ' means in the following that for any $j \in$ $\{1, \ldots, n\}$ and any $\zeta_{(j)}=\left(\zeta_{1}, \ldots, \zeta_{j-1}, \zeta_{j+1}, \ldots, \zeta_{n}\right) \in K_{\varepsilon}^{n-1}$, the function $\zeta_{j} \mapsto$ $p\left(\zeta_{1}, \ldots, \zeta_{n}\right)$ is analytic on the interior $K_{\varepsilon}^{\circ}$.

Definition 2.1. A function $p$ is an element of the set $S_{\mathrm{r}-\mathrm{a}}^{m, \varepsilon}$ if $p \in S^{m}, p$ is analytic on $K_{\varepsilon}^{n}$, there is a nonnegative $C$ such that

$$
|p(\zeta)| \leq C(1+|\operatorname{Re} \zeta|)^{m}, \quad \zeta \in K_{\varepsilon}^{n}
$$

and

$$
\sum_{\alpha \in \mathbb{N}_{0}^{n}}\|p\|_{\alpha}^{(m)} \varepsilon^{|\alpha|} / \alpha !<\infty \text {. Also, } S_{\mathrm{r}-\mathrm{a}}^{-\infty, \varepsilon}=\bigcap_{\mu \in \mathbb{R}} S_{\mathrm{r}-\mathrm{a}}^{\mu, \varepsilon}
$$

$S_{\mathrm{r}-\mathrm{a}}^{m, \varepsilon}$ is a vector subspace of the space $S^{m}$ of standard pseudodifferential symbols. The map taking each $p \in S_{\mathrm{r}-\mathrm{a}}^{m, \varepsilon}$ to the smallest number $C$ satisfying (2.2) is a norm on $S_{\mathrm{r}-\mathrm{a}}^{m, \varepsilon}$; write $\|\cdot\|_{*}^{(m)}$ for this norm and $\mathfrak{T}_{*}^{m}$ for the corresponding open ball topology on $S_{\mathrm{r}-\mathrm{a}}^{m, \varepsilon}$. The natural topology $\mathfrak{T}_{\mathrm{rel}}^{m}$ on $S_{\mathrm{r}-\mathrm{a}}^{m, \varepsilon}$ is that induced by $\mathfrak{T}^{m}$, and it is in particular generated by the sets

$$
\left\{p \in S_{\mathrm{r}-\mathrm{a}}^{m, \varepsilon},\|p-q\|_{\alpha}^{(m)}<t\right\}, \quad \alpha \in \mathbb{N}_{0}^{n}, t>0, q \in S^{m} .
$$

Lemma 2.2. $\mathfrak{T}_{\text {rel }}^{m}$ is stronger than $\mathfrak{T}_{*}^{m}$.

Proof. Fix $p \in S_{\mathrm{r}-\mathrm{a}}^{m, \varepsilon}$ and $\delta>0$. Given $\zeta \in K_{\varepsilon}^{n}$ with $\zeta_{j} \in[-R, R]$ for $j \in I \subseteq$ $\{1, \ldots, n\}$, and $\operatorname{Re} \zeta_{j}>R$ for $j \in J=\{1, \ldots, n\} \backslash I$, Taylor's formula implies, with $\xi=\operatorname{Re} \zeta$ and $\eta=\operatorname{Im} \zeta$,

$$
|p(\zeta)|=\left|\sum_{\alpha \in \mathbb{N}_{0}^{|J|}} \frac{1}{\alpha !} \partial_{\xi J}^{\alpha} p(\xi)\left(i \eta_{J}\right)^{\alpha}\right| \leq \sum_{\alpha \in \mathbb{N}_{0}^{n}} \frac{\|p\|_{\alpha}^{(m)}}{\alpha !}(1+|\xi|)^{m-|\alpha|} \varepsilon^{|\alpha|}(1+|\xi|)^{|\alpha|} .
$$

Since $\sum_{\alpha \in \mathbb{N}_{0}^{n}}\|p\|_{\alpha}^{(m)} \varepsilon^{|\alpha|} / \alpha !=\sum_{|\alpha| \leq \mu}\|p\|_{\alpha}^{(m)} \varepsilon^{|\alpha|} / \alpha !+\sum_{|\alpha|>\mu}\|p\|_{\alpha}^{(m)} \varepsilon^{|\alpha|} / \alpha !<\infty$ for $\mu \in \mathbb{N}_{0}$, there is a natural $\mu$ and a positive $t$ such that the open ball $\left\{\|p\|_{*}^{(m)}<\right.$ $\delta\} \in \mathfrak{T}_{*}^{m}$ includes the set

$$
\bigcap_{|\alpha| \leq \mu}\left\{\|p\|_{\alpha}^{(m)}<t\right\} \in \mathfrak{T}_{\mathrm{rel}}^{m}
$$

2.2. Real-analytic operators. Here we use the symbols in $S_{\mathrm{r}-\mathrm{a}}^{m, \varepsilon}$ to define a family of operators. The operators are expressed in terms of integrals over certain complex contours in $\mathbb{C}^{n} \times \mathbb{C}^{n}$, described first.

Let $a, a^{\prime}, b^{-}, b^{+}$and $c$ be $n$-tuples of elements of $\left.] 0, \infty\right]$ such that $a_{j}^{\prime}<c_{j}<a_{j}$ and $\max \left\{b_{j}^{-}, b_{j}^{+}\right\}<\varepsilon\left(c_{j}-a_{j}^{\prime}\right)$ for each $j=1, \ldots, n$. Let $\chi_{j}$ be a 'window function' in $C_{0}^{\infty}(\mathbb{R})$ satisfying

$$
\begin{gathered}
\chi_{j}(t)=1, \quad|t| \leq c_{j}, \\
\left.\chi_{j}(t) \in\right] 0,1\left[, \quad c_{j}<|t|<a_{j},\right. \\
\chi_{j}(t)=0, \quad|t| \geq a_{j} .
\end{gathered}
$$


With $R$ a positive constant, let $\psi \in C^{\infty}(\mathbb{R})$ be an even 'excision function' satisfying

$$
\begin{gathered}
\psi(t)=1, \quad|t| \geq 2 R, \\
\psi(t) \in] 0,1[, \quad R<|t|<2 R, \\
\psi(t)=0, \quad|t| \leq R .
\end{gathered}
$$

Also, let for $j=1, \ldots, n$ and for all real $t$ and $t^{\prime}$ :

$$
\begin{aligned}
& s_{j}\left(t, t^{\prime}\right)=-\chi_{j}(t) \psi\left(t^{\prime}\right) b_{j}^{-\operatorname{sgn} t^{\prime}} \operatorname{sgn} t^{\prime}, \\
& \eta_{j}\left(t, t^{\prime}\right)=\left(\chi_{j}(t)-1\right) \psi\left(t^{\prime}\right) b_{j}^{-\operatorname{sgn} t^{\prime}}\left|t^{\prime}\right| /\left(c_{j}-a_{j}^{\prime}\right) \operatorname{sgn} t .
\end{aligned}
$$

Fix an odd positive integer $N$, write

$$
s(y, \xi)=\left(s_{1}\left(y_{1}, \xi_{1}\right), \ldots, s_{n}\left(y_{n}, \xi_{n}\right)\right) \quad \text { and } \quad \eta(y, \xi)=\left(\eta_{1}\left(y_{1}, \xi_{1}\right), \ldots, \eta_{n}\left(y_{n}, \xi_{n}\right)\right)
$$

for all $y$ and $\xi$ in $\mathbb{R}^{n}$, introduce the map

$$
\sigma:[0,1] \times \mathbb{R}^{n} \times \mathbb{R}^{n} \rightarrow \mathbb{C}^{2 n}
$$

by

$$
\sigma(t, y, \xi)=\left(y+i t^{N} s(y, \xi), \xi+i t^{N} \eta(y, \xi)\right),
$$

and define the corresponding family of complex contours by

$$
\mathscr{C}_{t}=\sigma\left(t, \mathbb{R}^{n} \times \mathbb{R}^{n}\right), t \in[0,1] .
$$

As shown in the proof of Theorem 2.6 below, the mapping $\mathbb{R}^{n} \times \mathbb{R}^{n} \ni(y, \xi) \mapsto$ $\sigma(t, y, \xi)$ is orientation-preserving for each fixed $t \in[0,1]$. Every contour $\mathscr{C}_{t}$ is in the following given the orientation induced by its parametrisation $\sigma(t, \cdot, \cdot)$.

Remark 2.3. We construct the contours $\mathscr{C}_{t}$ such that the function $\mathbb{C}^{2 n} \ni(w, \zeta) \mapsto$ $\left|e^{i \zeta(z-w)}\right|$ is decreasing with respect to $\left|\xi_{j}\right|=\left|\operatorname{Re} \zeta_{j}\right|$ when $\left.t \in\right] 0,1\left[,(w, \zeta) \in \mathscr{C}_{t}\right.$, $z \in T\left(a^{\prime}, t^{N} b^{ \pm}\right)$and $\left|\xi_{j}\right| \geq 2 R$. Indeed, we have in this case that $\left(y_{j}-\operatorname{Re} z_{j}\right) \operatorname{sgn} y_{j} \mid$ $\left(c_{j}-a_{j}^{\prime}\right) \geq 1$ when $\chi_{j}\left(y_{j}\right)-1 \neq 0$, as well as that $\psi\left(\xi_{j}\right)=1$, so

$$
\begin{aligned}
& \operatorname{Re} i \zeta_{j}\left(z_{j}-w_{j}\right)=\left(t^{N} s_{j}-\operatorname{Im} z_{j}\right) \xi_{j}+\left(y_{j}-\operatorname{Re} z_{j}\right) t^{N} \eta_{j} \\
& \quad=-\left|\xi_{j}\right|\left(\operatorname{Im} z_{j} \operatorname{sgn} \xi_{j}+t^{N} \chi_{j} b_{j}^{-\operatorname{sgn} \xi_{j}}\right)+t^{N}\left|\xi_{j}\right| b_{j}^{-\operatorname{sgn} \xi_{j}}\left(\chi_{j}-1\right) \frac{y_{j}-\operatorname{Re} z_{j}}{c_{j}-a_{j}^{\prime}} \operatorname{sgn} y_{j} \\
& \quad \leq-\left|\xi_{j}\right|\left(\operatorname{Im} z_{j} \operatorname{sgn} \xi_{j}+t^{N} b_{j}^{-\operatorname{sgn} \xi_{j}}\right),
\end{aligned}
$$

and it is readily verified that $\operatorname{Im} z_{j} \operatorname{sgn} \xi_{j}+t^{N} b_{j}^{-\operatorname{sgn} \xi_{j}}>0$. Note that, furthermore, $\chi_{j}\left(y_{j}\right)=1$ implies $\operatorname{Re} i \zeta_{j}\left(z_{j}-w_{j}\right)=-\left|\xi_{j}\right|\left(\operatorname{Im} z_{j} \operatorname{sgn} \xi_{j}+t^{N} b_{j}^{-\operatorname{sgn} \xi_{j}}\right)$, which is negative only if $\left.\operatorname{Im} z_{j} \in\right]-t^{N} b_{j}^{-}, t^{N} b_{j}^{+}$. Finally, if $\chi_{j}\left(y_{j}\right)<1$ and $\operatorname{Im} z_{j}=0$, then $\operatorname{Re} i \zeta_{j}\left(z_{j}-w_{j}\right)=-\left|\xi_{j}\right| t^{N} b_{j}^{-\operatorname{sgn} \xi_{j}}\left(\chi_{j}+\left(1-\chi_{j}\right)\left(y_{j}-z_{j}\right) \operatorname{sgn} y_{j} /\left(c_{j}-a_{j}^{\prime}\right)\right)$, which is negative if and only if $\left.z_{j} \in\right]-c_{j}, c_{j}[$.

The real-analytic operators of this section are constructed to map to and from the following spaces of functions.

Definition 2.4. The space $\mathscr{O}\left(a^{\prime}, b^{ \pm}\right)$consists precisely of those functions that are analytic on $T\left(a^{\prime}, b^{ \pm}\right)$. The elements of the space $\widetilde{\mathscr{O}}\left(a, b^{ \pm}\right)$are functions that are analytic on $T\left(a, b^{ \pm}\right), C^{1}$ w.r.t. $\operatorname{Re} z_{j}$ and $\operatorname{Im} z_{j}$ on the closure $\overline{T\left(a, b^{ \pm}\right)}$and that vanish on $\mathbb{R}^{n} \backslash \overline{\tau(a)}$. 
We equip the space $\mathscr{O}\left(a^{\prime}, b^{ \pm}\right)$with the topology of uniform convergence on compact subsets of $T\left(a^{\prime}, b^{ \pm}\right)$, and the space $\widetilde{\mathscr{O}}\left(a, b^{ \pm}\right)$with the topology induced by the usual $C^{1, \alpha}$ norm

$$
\|u\|=\sum_{\substack{\beta \in \mathbb{N}_{0}^{2 n} \\|\beta| \leq 1}}\left(\left\|\partial_{\operatorname{Re} z, \operatorname{Im} z}^{\beta} u\right\|_{L^{\infty}\left(\overline{T\left(a, b^{ \pm}\right)}\right)}+\sup _{T\left(a, b^{ \pm}\right)} \frac{\left|\partial_{\operatorname{Re} z, \operatorname{Im} z}^{\beta} u(x)-\partial_{\operatorname{Re} z, \operatorname{Im} z}^{\beta} u(y)\right|}{|x-y|^{\alpha}}\right)
$$

for some $\alpha \in] 0,1\left[\right.$. It is well-known that $\mathscr{O}\left(a^{\prime}, b^{ \pm}\right)$is then Fréchet and $\widetilde{\mathscr{O}}\left(a, b^{ \pm}\right)$is Banach. The spaces

$$
\mathscr{L}\left(\widetilde{\mathscr{O}}\left(a, b^{ \pm}\right), L^{\infty}(\tau(c))\right) \quad \text { and } \quad \mathscr{L}\left(\widetilde{\mathscr{O}}\left(a, b^{ \pm}\right), \mathscr{O}\left(a^{\prime}, b^{ \pm}\right)\right)
$$

of continuous linear mappings are equipped with the corresponding topologies of bounded convergence and are thus made locally convex (see pp. 131-133 of Köthe [12.) In particular, the topologies on these spaces are defined by the systems of seminorms

$$
\|Q\|_{W}=\sup _{u \in W}\|Q u\|_{L^{\infty}(\tau(c))}
$$

and

$$
\|Q\|_{W, \kappa}=\sup _{u \in W} \sup _{z \in \kappa}|Q u(z)|,
$$

respectively, where $W$ is a bounded subset of $\widetilde{\mathscr{O}}\left(a, b^{ \pm}\right)$and $\kappa$ is a compact subset of $T\left(a^{\prime}, b^{ \pm}\right)$. Since $\widetilde{\mathscr{O}}\left(a, b^{ \pm}\right)$is normed and $L^{\infty}(\tau(c))$ is Banach, $\mathscr{L}\left(\widetilde{\mathscr{O}}\left(a, b^{ \pm}\right), L^{\infty}(\tau(c))\right)$ is complete.

Definition 2.5. For every symbol $p$ in $S_{\mathrm{r}-\mathrm{a}}^{m, \varepsilon}, \operatorname{OP}(p)$ is the operator on $\widetilde{\mathscr{O}}\left(a, b^{ \pm}\right)$ given by

$$
\mathrm{OP}(p) u(z)=(2 \pi)^{-n} \int_{(w, \zeta) \in \mathscr{C}_{1}} e^{i \zeta(z-w)} p(\zeta) u(w), \quad z \in T\left(a^{\prime}, b^{ \pm}\right), u \in \widetilde{\mathscr{O}}\left(a, b^{ \pm}\right) .
$$

Theorem 2.6. The maps

$$
\mathrm{OP}: S_{\mathrm{r}-\mathrm{a}}^{m, \varepsilon} \rightarrow \mathscr{L}\left(\widetilde{\mathscr{O}}\left(a, b^{ \pm}\right), L^{\infty}(\tau(c))\right)
$$

and

$$
\text { OP }: S_{\mathrm{r}-\mathrm{a}}^{m, \varepsilon} \rightarrow \mathscr{L}\left(\widetilde{\mathscr{O}}\left(a, b^{ \pm}\right), \mathscr{O}\left(a^{\prime}, b^{ \pm}\right)\right)
$$

are linear and continuous when $S_{\mathrm{r}-\mathrm{a}}^{m, \varepsilon}$ is equipped with the natural topology $\mathfrak{T}_{\mathrm{rel}}^{m}$.

Furthermore, if $p \in S_{\mathrm{r}-\mathrm{a}}^{-\infty, \varepsilon}, u \in \widetilde{\mathscr{O}}\left(a, b^{ \pm}\right)$and $x \in \tau\left(a^{\prime}\right)$, then

$$
\operatorname{OP}(p) u(x)=(2 \pi)^{-n} \int_{(y, \xi) \in \mathscr{C}_{0}} e^{i \xi(x-y)} p(\xi) u(y) .
$$

Proof. Let $\delta^{\mathrm{Kr}}$ be the 'Kronecker delta'; that is, for all integers $j, l$ set $\delta_{j, l}^{\mathrm{Kr}}=0$ if $j \neq l$ and $\delta_{j, l}^{\mathrm{Kr}}=1$ if $j=l$. For all $j, l=1, \ldots, n$ and all $\left.\left.(t, y, \xi) \in\right] 0,1\right] \times \mathbb{R}^{n} \times \mathbb{R}^{n}$, we have

$$
\begin{cases}\partial_{t} \sigma_{l}(t, y, \xi) & =-i N t^{N-1} \chi_{l}\left(y_{l}\right) \psi\left(\xi_{l}\right) b_{l}^{-\operatorname{sgn} \xi_{l}} \operatorname{sgn} \xi_{l} \\ \partial_{t} \sigma_{l+n}(t, y, \xi) & =i N t^{N-1}\left(\chi_{l}\left(y_{l}\right)-1\right) \psi\left(\xi_{l}\right) b_{l}^{-\operatorname{sgn} \xi_{l}}\left|\xi_{l}\right| \operatorname{sgn} y_{l} /\left(c_{l}-a_{l}^{\prime}\right)\end{cases}
$$




$$
\begin{cases}\partial_{y_{j}} \sigma_{l}(t, y, \xi) & =\delta_{j, l}^{\mathrm{Kr}}\left(1-i t^{N} \chi_{j}^{\prime}\left(y_{j}\right) \psi\left(\xi_{j}\right) b_{j}^{-\operatorname{sgn} \xi_{j}} \operatorname{sgn} \xi_{j}\right) \\ \partial_{y_{j}} \sigma_{l+n}(t, y, \xi) & =i t^{N} \delta_{j, l}^{\mathrm{Kr}} \chi_{j}^{\prime}\left(y_{j}\right) \psi\left(\xi_{j}\right) b_{j}^{-\operatorname{sgn} \xi_{j}}\left|\xi_{j}\right| \operatorname{sgn} y_{j} /\left(c_{j}-a_{j}^{\prime}\right)\end{cases}
$$

and

$$
\left\{\begin{array}{l}
\partial_{\xi_{j}} \sigma_{l}(t, y, \xi)=-i t^{N} \delta_{j, l}^{\mathrm{Kr}} \chi_{j}\left(y_{j}\right) \psi^{\prime}\left(\xi_{j}\right) b_{j}^{-\operatorname{sgn} \xi_{j}} \operatorname{sgn} \xi_{j}, \\
\partial_{\xi_{j}} \sigma_{l+n}(t, y, \xi)=\delta_{j, l}^{\mathrm{Kr}}\left(1+i t^{N}\left(\chi_{j}\left(y_{j}\right)-1\right) b_{j}^{-\operatorname{sgn} \xi_{j}} \operatorname{sgn} y_{j} \frac{\psi^{\prime}\left(\xi_{j}\right)\left|\xi_{j}\right|+\psi\left(\xi_{j}\right) \operatorname{sgn} \xi_{j}}{c_{j}-a_{j}^{\prime}}\right) .
\end{array}\right.
$$

Thus, for each $t \in] 0,1]$, the Jacobi determinant of the mapping $(y, \xi) \mapsto \sigma(t, y, \xi)$ is at most polynomially increasing with respect to $\left|\xi_{1}\right|, \ldots,\left|\xi_{n}\right|$, and, in view of Remark 2.3. the function $(w, \zeta) \mapsto e^{i \zeta(z-w)} p(\zeta) u(w)$ is absolutely integrable over the contour $\mathscr{C}_{t}$ when $z \in \tau(c)$ or $z \in T\left(a^{\prime}, t^{N} b^{ \pm}\right)$, as well as $\left.\left.t \in\right] 0,1\right], p \in S_{\mathrm{r}-\mathrm{a}}^{m, \varepsilon}$ and $u \in \widetilde{\mathscr{O}}\left(a, b^{ \pm}\right)$. In particular, we have for all $p \in S_{\mathrm{r}-\mathrm{a}}^{m, \varepsilon}$ and $u \in \widetilde{\mathscr{O}}\left(a, b^{ \pm}\right)$that

$$
|\mathrm{OP}(p) u(z)| \leq C(z)\|p\|_{*}^{(m)}\|u\|, \quad z \in \tau(c) \cup T\left(a^{\prime}, b^{ \pm}\right),
$$

where

$$
C(z)=\int_{(y, \xi) \in \tau(a) \times \mathbb{R}^{n}}(1+|\xi|)^{m}\left|e^{i \zeta(z-w)} \operatorname{det} d_{y, \xi} \sigma(1, y, \xi)\right|, \quad z \in \tau(c) \cup T\left(a^{\prime}, b^{ \pm}\right),
$$

is a continuous function independent of $p$ and $u$ (see part (a) of Theorem (2.27) on p. 54 in Folland [4]). Thus the linear map $\mathrm{OP}(p): \widetilde{\mathscr{O}}\left(a, b^{ \pm}\right) \rightarrow L^{\infty}(\tau(c))$ is continuous when $p \in S_{\mathrm{r}-\mathrm{a}}^{m, \varepsilon}$. Also, there is a nonnegative constant $C$ such that, for all $p \in S_{\mathrm{r}-\mathrm{a}}^{m, \varepsilon}$ and every bounded subset $W$ of $\widetilde{\mathscr{O}}\left(a, b^{ \pm}\right)$,

$$
\sup _{u \in W}\|\mathrm{OP}(p) u\|_{L^{\infty}(\tau(c))} \leq\left(C \sup _{u \in W}\|u\|\right)\|p\|_{*}^{(m)},
$$

so the linear map OP $: S_{\mathrm{r}-\mathrm{a}}^{m, \varepsilon} \rightarrow \mathscr{L}\left(\widetilde{\mathscr{O}}\left(a, b^{ \pm}\right), L^{\infty}(\tau(c))\right)$ is continuous w.r.t. the open ball topology $\mathfrak{T}_{*}^{m}$ on $S_{\mathrm{r}-\mathrm{a}}^{m, \varepsilon}$, and hence it is also continuous w.r.t. the stronger topology $\mathfrak{T}_{\text {rel }}^{m}$.

For each $j=1, \ldots, n$ and each $z \in T\left(a^{\prime}, b^{ \pm}\right)$we have (see part (b) of Theorem $(2.27)$ in [4])

$$
(2 \pi)^{n} \partial_{\bar{z}_{j}} \mathrm{OP}(p) u(z)=\partial_{\bar{z}_{j}} \int_{\mathscr{C}_{1}} e^{i \zeta(z-w)} p(\zeta) u(w)=\int_{\mathscr{C}_{1}} \partial_{\bar{z}_{j}} e^{i \zeta(z-w)} p(\zeta) u(w)=0 .
$$

Furthermore, for every $p \in S_{\mathrm{r}-\mathrm{a}}^{m, \varepsilon}$ and every compact subset $\kappa$ of $T\left(a^{\prime}, b^{ \pm}\right)$, there exists a positive $C_{\kappa}$, depending only on $\kappa$, such that

$$
\sup _{z \in \kappa}|\mathrm{OP}(p) u(z)| \leq\left(C_{\kappa}\|p\|_{*}^{(m)}\right)\|u\|
$$

for all $u \in \widetilde{\mathscr{O}}\left(a, b^{ \pm}\right)$, and the linear map $\mathrm{OP}(p): \widetilde{\mathscr{O}}\left(a, b^{ \pm}\right) \rightarrow \mathscr{O}\left(a^{\prime}, b^{ \pm}\right)$is continuous. Finally, for every bounded subset $W$ of $\widetilde{\mathscr{O}}\left(a, b^{ \pm}\right)$and every compact subset $\kappa$ of $T\left(a^{\prime}, b^{ \pm}\right)$we have

$$
\sup _{u \in W} \sup _{z \in \kappa}|\mathrm{OP}(p) u(z)| \leq\left(C_{\kappa} \sup _{u \in W}\|u\|\right)\|p\|_{*}^{(m)}, \quad p \in S_{\mathrm{r}-\mathrm{a}}^{m, \varepsilon}
$$

so the linear map

$$
\mathrm{OP}: S_{\mathrm{r}-\mathrm{a}}^{m, \varepsilon} \rightarrow \mathscr{L}\left(\widetilde{\mathscr{O}}\left(a, b^{ \pm}\right), \mathscr{O}\left(a^{\prime}, b^{ \pm}\right)\right)
$$

is continuous w.r.t. the topology $\mathfrak{T}_{*}^{m}$, and hence also w.r.t. $\mathfrak{T}_{\text {rel }}^{m}$. This finishes the proof of the first part of Theorem 2.6 
We now prove the validity of (2.4) using Stokes's theorem. Fix $\rho>R$ and write

$$
\left.D_{\rho}=\right] 0,1\left[\times \tau(a) \times(]-\rho, \rho{ }^{n} \backslash[-R, R]^{n}\right),
$$

as well as $M_{\rho}=\sigma\left(D_{\rho}\right)$. The set $M_{\rho}$ is included in $T\left(a, b^{ \pm}\right) \times K_{\varepsilon}^{n}$, since for each $t \in] 0,1\left[, j=1, \ldots, n, y_{j} \in \mathbb{R}\right.$ and $\xi_{j} \in \mathbb{R}$ we have $t^{N} s_{j}\left(y_{j}, \xi_{j}\right) \in\left[-t^{N} b_{j}^{-}, t^{N} b_{j}^{+}\right] \subset$ ]$-b_{j}^{-}, b_{j}^{+}\left[\right.$, as well as $\left|t^{N} \eta_{j}\left(y_{j}, \xi_{j}\right)\right| \leq t^{N}\left|\psi\left(\xi_{j}\right)\right| \cdot\left|\xi_{j}\right| b_{j}^{-\operatorname{sgn} \xi_{j}} /\left(c_{j}-a_{j}^{\prime}\right)$, with $b_{j}^{-\operatorname{sgn} \xi_{j}} /$ $\left(c_{j}-a_{j}^{\prime}\right)<\varepsilon$. The closure $\bar{D}_{\rho}$ is a compact, smooth and oriented $(2 n+1)$ manifold with corners, and the map $\sigma$ is smooth and injective on an open neighbourhood of $\bar{D}_{\rho}$. The injectivity follows from the fact that the functions $s$ and $\eta$ are real-valued, and $N$ is odd. Indeed, if $y+i t^{N} s(y, \xi)=\widetilde{y}+i \widetilde{t}^{N} s(\widetilde{y}, \widetilde{\xi})$ and $\xi+i t^{N} \eta(y, \xi)=\widetilde{\xi}+i \widetilde{t}{ }^{N} \eta(\widetilde{y}, \widetilde{\xi})$, then $y=\widetilde{y}$ and $\xi=\widetilde{\xi}$, and hence $t=\widetilde{t}$. It is here of interest to study the rank of $\sigma$ and of some of its restrictions. The matrices $\left(\operatorname{Re} \partial_{y_{j}} \sigma_{l}\right)_{l, j}$ and $\left(\operatorname{Re} \partial_{\xi_{j}} \sigma_{l+n}\right)_{l, j}$ are the identity matrix, and the matrices $\left(\operatorname{Re} \partial_{\xi_{j}} \sigma_{l}\right)_{l, j}$ and $\left(\operatorname{Re} \partial_{y_{j}} \sigma_{l+n}\right)_{l, j}$ are the zero matrix, so for every $t$ in an open neighbourhood of $[0,1]$ the Jacobian of the map $(y, \xi) \mapsto \sigma(t, y, \xi)$ has rank $2 n$, and each contour $\mathscr{C}_{t, \rho}=\sigma(t, \tau(a) \times]-\rho, \rho\left[{ }^{n}\right), t \in[0,1]$, is a $2 n$-dimensional immersed smooth submanifold of $\mathbb{C}^{2 n}$. For each $(t, y, \xi)$ in $D_{\rho}$, the quantity $\partial_{t} \sigma(t, y, \xi)$ is nonzero and purely imaginary, since $\left|\xi_{l}\right|>R$ for some $l=1, \ldots, n$, and since $\chi_{l}\left(y_{l}\right)$ and $\chi_{l}\left(y_{l}\right)-1$ are never both zero. Thus, the Jacobian of the map $\sigma$ has full rank on $D_{\rho}$, so $\sigma$ is an injective immersion there and the set $M_{\rho}$ is a smooth, $(2 n+1)$-dimensional immersed submanifold of $\mathbb{C}^{2 n}$. Similarly, each element of the contour

$$
\mathscr{D}_{j, \rho}^{ \pm}=\sigma(] 0,1\left[\times \tau(a) \times\left\{\xi_{l} \in\right]-\rho, \rho\left[\text { for } l \neq j, \xi_{j}= \pm \rho\right\}\right), \quad j=1, \ldots, n,
$$

is a $2 n$-dimensional immersed smooth submanifold of $\mathbb{C}^{2 n}$.

The map $\sigma$ induces an orientation on $M_{\rho}$, where the ordered basis of $T M_{\rho}$ given by $\left(d \sigma \frac{d}{d t}, d \sigma \partial_{y_{1}}, \ldots, d \sigma \partial_{y_{n}}, d \sigma \partial_{\xi_{1}}, \ldots, d \sigma \partial_{\xi_{n}}\right)$ is positively oriented. The restrictions of $\sigma$ to the sets $\sigma^{-1}\left(\mathscr{C}_{1, \rho}\right), \sigma^{-1}\left(\mathscr{D}_{j, \rho}^{+}\right)$with $j+n$ even, as well as $\sigma^{-1}\left(\mathscr{D}_{j, \rho}^{-}\right)$ with $j+n$ odd, preserve the Stokes orientation of the corresponding contours $\mathscr{C}_{1, \rho}$, $\mathscr{D}_{j, \rho}^{+}(j+n$ even $)$ and $\mathscr{D}_{j, \rho}^{-}(j+n$ odd $)$. The restrictions of $\sigma$ to the sets $\sigma^{-1}\left(\mathscr{C}_{0, \rho}\right)$, $\sigma^{-1}\left(\mathscr{D}_{j, \rho}^{+}\right)$with $j+n$ odd, as well as $\sigma^{-1}\left(\mathscr{D}_{j, \rho}^{-}\right)$with $j+n$ even, reverse the Stokes orientation of the corresponding contours $\mathscr{C}_{0, \rho}, \mathscr{D}_{j, \rho}^{+}(j+n$ odd $)$ and $\mathscr{D}_{j, \rho}^{-}(j+n$ even $)$. Furthermore, there is an open neighbourhood $\mathscr{N}$ of $\overline{\tau(a)} \times\left([-\rho, \rho]^{n} \backslash\right]-R, R\left[^{n}\right)$ such that, for all $j=1, \ldots, n$,

$$
\begin{aligned}
d \sigma_{j} & =i N t^{N-1} s_{j} d t+\left(1+i t^{N} \partial_{y_{j}} s_{j}\right) d y_{j}+i t^{N} \partial_{\xi_{j}} s_{j} d \xi_{j} \quad \text { and } \\
d \sigma_{n+j} & =i N t^{N-1} \eta_{j} d t+i t^{N} \partial_{y_{j}} \eta_{j} d y_{j}+\left(1+i t^{N} \partial_{\xi_{j}} \eta_{j}\right) d \xi_{j}
\end{aligned}
$$

when $(t, y, \xi) \in] 0,1\left[\times \mathscr{N}\right.$. The restrictions of the forms $d y_{j}$ and $d \xi_{j}$ to those subsets of $\partial D_{\rho}$ where $y_{j}$ or $\xi_{j}$ is constant, respectively, are identically zero. Since $s_{j}\left(y_{j}, \xi_{j}\right)$ and $\partial_{\xi_{j}} s_{j}\left(y_{j}, \xi_{j}\right)$ equal zero when $\left|y_{j}\right|=a_{j}$, the restriction of $d \sigma_{j}$ to any subset of $\partial D_{\rho}$ where $\left.t \in\right] 0,1\left[\right.$ and $\left|y_{j}\right|=a_{j}$ is identically zero. Also, since $\eta_{j}\left(y_{j}, \xi_{j}\right)$ and $\partial_{y_{j}} \eta_{j}\left(y_{j}, \xi_{j}\right)$ equal zero when $\left|\xi_{j}\right|=R$, the restriction of $d \sigma_{n+j}$ to any subset of $\partial D_{\rho}$ where $\left.t \in\right] 0,1\left[\right.$ and $\left|\xi_{j}\right|=R$ is identically zero. Finally, each component of the boundary $\partial D_{\rho}$ where two or more of the quantities $t, y_{1}, \ldots, y_{n}, \xi_{1}, \ldots, \xi_{n}$ are constant has dimension less than $2 n$, so restrictions of differential $2 n$-forms to such components of $\partial D_{\rho}$ are identically zero. 
Now fix $p \in S_{\mathrm{r}-\mathrm{a}}^{-\infty, \varepsilon}, u \in \widetilde{\mathscr{O}}\left(a, b^{ \pm}\right)$and $x \in \tau\left(a^{\prime}\right)$. The function

$$
G(w, \zeta)=e^{i \zeta(x-w)} p(\zeta) u(w)
$$

is analytic on $T\left(a, b^{ \pm}\right) \times K_{\varepsilon}^{n}$, so the associated complex $2 n$-form

$$
\mu=G d w_{1} \wedge \cdots \wedge d w_{n} \wedge d \zeta_{1} \wedge \cdots \wedge d \zeta_{n}=G d w \wedge d \zeta
$$

is closed there:

$$
d \mu=\sum_{j=1}^{n}\left(\partial_{w_{j}} G d w_{j}+\partial_{\bar{w}_{j}} G d \bar{w}_{j}+\partial_{\zeta_{j}} G d \zeta_{j}+\partial_{\bar{\zeta}_{j}} G d \bar{\zeta}_{j}\right) d w \wedge d \zeta=0 .
$$

Also, the form $\sigma^{*} \mu=(G \circ \sigma) d \sigma_{1} \wedge \cdots \wedge d \sigma_{2 n}$ is $C^{1}$ on the closure $\bar{D}_{\rho}$, so

$$
\int_{M_{\rho}} d \mu=\int_{D_{\rho}} \sigma^{*} d \mu=\int_{D_{\rho}} d \sigma^{*} \mu=\int_{\partial D_{\rho}} \sigma^{*} \mu,
$$

where the last equality follows from Stokes's theorem on manifolds with corners, given, e.g., as Theorem 14.20 on p. 367 in Lee [13. Since $\mathscr{C}_{0, \rho}$ and $\mathscr{C}_{1, \rho}$ both include the set $\tau(a) \times[-R, R]^{n}$ but have mutually opposite Stokes orientation, (2.8) implies

$$
\int_{\mathscr{C}_{1, \rho}} \mu-\int_{\mathscr{C}_{0, \rho}} \mu+\sum_{\substack{1 \leq j \leq n \\ j+n \text { even }}}\left(\int_{\mathscr{D}_{j, \rho}^{+}} \mu-\int_{\mathscr{D}_{j, \rho}^{-}} \mu\right)+\sum_{\substack{1 \leq j \leq n \\ j+n \text { odd }}}\left(\int_{\mathscr{D}_{j, \rho}^{-}} \mu-\int_{\mathscr{D}_{j, \rho}^{+}} \mu\right)=0 .
$$

For $t \in\{0,1\}$ we have

$$
\begin{aligned}
\int_{\mathscr{C}_{t, \rho}} \mu & =\int_{\mathscr{C}_{0, \rho}} \sigma(t, \cdot)^{*} \mu=\int_{(y, \xi) \in \mathscr{C}_{0, \rho}} G \circ \sigma(t, y, \xi) \operatorname{det}\left(d_{y, \xi} \sigma(t, y, \xi)\right) d y \wedge d \xi \\
& =\int_{(y, \xi) \in \mathscr{C}_{0, \rho}} G \circ \sigma(t, y, \xi) \operatorname{det}\left(d_{y, \xi} \sigma(t, y, \xi)\right)=\int_{\mathscr{C}_{t, \rho}} G .
\end{aligned}
$$

The function $G$ is absolutely integrable over $\mathscr{C}_{0}$ since $\mathscr{C}_{0}=\mathbb{R}^{n} \times \mathbb{R}^{n}, p \in S^{-\infty}$, and $u \in L^{\infty}\left(\mathbb{R}^{n}\right)$ is compactly supported. In view of the discussion near the beginning of this proof, the function $G$ is thus absolutely integrable over each contour $\mathscr{C}_{t}$, $t \in[0,1]$. Therefore, in particular,

$$
\lim _{\rho \rightarrow \infty} \int_{\mathscr{C}_{0, \rho}} G=\int_{\mathscr{C}_{0}} G \quad \text { and } \quad \lim _{\rho \rightarrow \infty} \int_{\mathscr{C}_{1, \rho}} G=\int_{\mathscr{C}_{1}} G .
$$

It remains to estimate the integrals over the contours $\mathscr{D}_{j, \rho}^{ \pm}$in (2.9). Using repeated integration by parts, we first note that, for each $\nu \in \mathbb{N}_{0}$ and $\rho>1$,

$$
\int_{t=0}^{1} t^{N-1+\nu} e^{-t^{N} \rho b_{j}^{-\operatorname{sgn} \xi_{j}}}=O\left(\rho^{-N-\nu}\right) .
$$

As seen from (2.5)-(2.7), the Jacobi determinant of the restriction of $\sigma$ to $\sigma^{-1}\left(\mathscr{D}_{j, \rho}^{ \pm}\right)$ is a polynomial in the variables $t,\left|\xi_{1}\right|, \ldots,\left|\xi_{j-1}\right|,\left|\xi_{j+1}\right|, \ldots,\left|\xi_{n}\right|$. The first column of the Jacobian is proportional to $t^{N-1}$, so each term of the determinant is of order at least $N-1$ w.r.t. $t$. Write $M^{\prime}$ for the maximal order of the Jacobi determinant w.r.t. the variables $\left(\left|\xi_{1}\right|, \ldots,\left|\xi_{j-1}\right|,\left|\xi_{j+1}\right|, \ldots,\left|\xi_{n}\right|\right)$. There are constants

$$
\begin{gathered}
M \in \mathbb{N}_{0}, \\
C^{\prime}, C^{\prime \prime} \in \mathbb{R} \text {, and } \\
C_{\nu, \alpha} \in \mathbb{R} \text { for } \nu=0, \ldots, M, \alpha \in \mathbb{N}_{0}^{n-1},|\alpha| \leq M^{\prime},
\end{gathered}
$$


such that for all $\rho>1$

$$
\begin{aligned}
& \left|\int_{\mathscr{D}_{j, \rho}^{ \pm}} \mu\right| \leq \sum_{\nu=0}^{M} \sum_{\substack{\alpha \in \mathbb{N}_{0}^{n-1} \\
|\alpha| \leq M^{\prime}}} C_{\nu, \alpha} \rho^{|\alpha|} \int_{\sigma^{-1}\left(\mathscr{D}_{j, \rho}^{ \pm}\right)} t^{N-1+\nu}|G \circ \sigma| \\
& \leq C^{\prime}\|p\|_{0}^{(m)}\|u\| \rho^{M^{\prime}}(1+\rho \sqrt{n})^{|m|} \sum_{\nu=0}^{M} \int_{t=0}^{1} t^{N-1+\nu} e^{-t^{N} \rho b_{j}^{-\operatorname{sgn} \xi_{j}}} \\
& \times \prod_{l \neq j} \int_{\xi_{l}=-\rho}^{\rho} e^{-t^{N}\left|\xi_{l}\right| b_{l}^{-\operatorname{sgn} \xi_{l}} \psi\left(\xi_{l}\right)} \\
& \leq C^{\prime \prime} \rho^{M^{\prime}+|m|} \sum_{\nu=0}^{M} \int_{t=0}^{1} t^{N-1+\nu} e^{-t^{N} \rho b_{j}^{-\operatorname{sgn} \xi_{j}}} \cdot(2 \rho)^{n-1} \\
& =O\left(\rho^{M^{\prime}+|m|+n-1-N}\right),
\end{aligned}
$$

and, in conclusion, $\lim _{\rho \rightarrow \infty} \int_{\mathscr{D}_{j, \rho}^{ \pm}} \mu=0$ for sufficiently large $N$. This completes the proof of the second part of Theorem 2.6 .

For $p \in S^{-\infty}$, the operator $\mathrm{P}(p)$ is defined on $\widetilde{\mathscr{O}}\left(a, b^{ \pm}\right)$by

$$
\mathrm{P}(p) u(x)=(2 \pi)^{-n} \iint_{(y, \xi) \in \mathbb{R}^{n} \times \mathbb{R}^{n}} e^{i \xi(x-y)} p(\xi) u(y), \quad x \in \mathbb{R}^{n}, u \in \widetilde{\mathscr{O}}\left(a, b^{ \pm}\right) .
$$

The mapping $\mathrm{P}: S_{\mathrm{r}-\mathrm{a}}^{-\infty, \varepsilon} \rightarrow \mathscr{L}\left(\widetilde{\mathscr{O}}\left(a, b^{ \pm}\right), L^{\infty}\left(\tau\left(a^{\prime}\right)\right)\right)$ is linear and continuous. Indeed, there is a nonnegative $C$ such that

$$
|\mathrm{P}(p) u(x)| \leq\|p\|_{0}^{(-n-1)}\|u\| \int_{y \in \tau(a)} \int_{\xi \in \mathbb{R}^{n}}(1+|\xi|)^{-n-1} \leq C\|p\|_{0}^{(-n-1)}\|u\|
$$

for all $p \in S_{\mathrm{r}-\mathrm{a}}^{-\infty, \varepsilon}, u \in \widetilde{\mathscr{O}}\left(a, b^{ \pm}\right)$and $x \in \tau\left(a^{\prime}\right)$.

Lemma 2.7. OP is the unique extension of $\mathrm{P}$ that is linear and continuous as a map

$$
\mathrm{OP}: S_{\mathrm{r}-\mathrm{a}}^{m, \varepsilon} \rightarrow \mathscr{L}\left(\widetilde{\mathscr{O}}\left(a, b^{ \pm}\right), L^{\infty}\left(\tau\left(a^{\prime}\right)\right)\right)
$$

for every fixed real $m$.

Proof. We first show the uniqueness of the extension. Pick $p \in S_{\mathrm{r}-\mathrm{a}}^{\mu, \varepsilon}$ for some real $\mu$, and consider the family of functions $f_{\lambda}(\zeta)=e^{-(\lambda \zeta)^{2}}, \zeta \in K_{\varepsilon}^{n}$, with $\lambda \geq 0$. Each $f_{\lambda}$ is analytic on $K_{\varepsilon}^{n}$. Also, $\left|f_{\lambda}(\zeta)\right| \leq \exp \left(-\lambda^{2}\left(1-\varepsilon^{2}\right)|\operatorname{Re} \zeta|^{2}\right)$ on $K_{\varepsilon}^{n}$, so, for $\lambda>0$, we have $f_{\lambda} \in S_{\mathrm{r}-\mathrm{a}}^{-\infty, \varepsilon}$ and $f_{\lambda} p \in S_{\mathrm{r}-\mathrm{a}}^{-\infty, \varepsilon}$. Since $f_{1} \in S^{0}$, it follows from Proposition 18.1.2 on page 66 of Hörmander [6] that $\lim _{\lambda \searrow 0} f_{\lambda}=f_{0}=1$ in $S^{t}$ for every positive $t$, so $\lim _{\lambda \searrow 0} f_{\lambda} p=p$ in $S^{\mu+t}$ for $t>0$. Now if $\mathrm{P}_{1}^{\prime}$ and $\mathrm{P}_{2}^{\prime}$ are extensions of $\mathrm{P}$ as described above, then $\left(\mathrm{P}_{1}^{\prime}-\mathrm{P}_{2}^{\prime}\right) p=\left(\mathrm{P}_{1}^{\prime}-\mathrm{P}_{2}^{\prime}\right) \lim _{\lambda \searrow 0} f_{\lambda} p=\lim _{\lambda \searrow 0}\left(\mathrm{P}_{1}^{\prime}-\mathrm{P}_{2}^{\prime}\right) f_{\lambda} p=0$, where $\lim _{\lambda \searrow 0} f_{\lambda} p$ is understood w.r.t. the topology $\mathfrak{T}_{\text {rel }}^{\mu+t}$.

Next, $\tau\left(a^{\prime}\right) \subset \tau(c)$, so the first part of Theorem 2.6 implies that OP maps $S_{\mathrm{r}-\mathrm{a}}^{m, \varepsilon}$ continuously to $\mathscr{L}\left(\widetilde{\mathscr{O}}\left(a, b^{ \pm}\right), L^{\infty}\left(\tau\left(a^{\prime}\right)\right)\right)$ for each real $m$. Finally, the second part of that theorem implies that $\mathrm{P}(p) u=\mathrm{OP}(p) u$ in $L^{\infty}\left(\tau\left(a^{\prime}\right)\right)$ when $p \in S_{\mathrm{r}-\mathrm{a}}^{-\infty, \varepsilon}$ and $u \in \widetilde{\mathscr{O}}\left(a, b^{ \pm}\right)$. 
Write $\operatorname{OPS}(p)$ for the standard pseudodifferential operator with symbol $p$, as defined in Section 18.1 of Hörmander [6]. Lemma 2.7 implies the following.

Corollary 2.8. If $p \in S_{\mathrm{r}-\mathrm{a}}^{m, \varepsilon}$ and $u \in \widetilde{\mathscr{O}}\left(a, b^{ \pm}\right)$, then $\operatorname{OPS}(p) u$ is the restriction to $\tau\left(a^{\prime}\right)$ of a function in $\mathscr{O}\left(a^{\prime}, b^{ \pm}\right)$.

Proof. The mapping OPS is by definition the unique linear continuous extension of the mapping $\mathrm{P}: S_{\mathrm{r}-\mathrm{a}}^{-\infty, \varepsilon} \rightarrow \mathscr{L}\left(\widetilde{\mathscr{O}}\left(a, b^{ \pm}\right), L^{\infty}\left(\tau\left(a^{\prime}\right)\right)\right)$ to $S_{\mathrm{r}-\mathrm{a}}^{m, \varepsilon}$. Thus, by Lemma 2.7 we have for all $p \in S_{\mathrm{r}-\mathrm{a}}^{m, \varepsilon}$ and all $u \in \widetilde{\mathscr{O}}\left(a, b^{ \pm}\right)$that $\sup _{x \in \tau\left(a^{\prime}\right)} \mid \operatorname{OPS}(p) u(x)-$ $\mathrm{OP}(p) u(x) \mid=0$. Finally, as shown in the first part of Theorem 2.6. $\operatorname{OP}(p) u \in$ $\mathscr{O}\left(a^{\prime}, b^{ \pm}\right)$.

Now follows the main result of this section. Let $f, g^{-}$and $g^{+}$be $n$-tuples of elements of $] 0, \infty]$, and assume $p \in S_{\mathrm{r}-\mathrm{a}}^{m, \varepsilon}$.

Theorem 2.9. If $u \in \widetilde{\mathscr{O}}\left(f, g^{ \pm}\right)$, then $\operatorname{OPS}(p) u$ is analytically continuable to each complex polyrectangle $T\left(\widetilde{a}, \widetilde{b}^{ \pm}\right)$with $\left.\widetilde{a}_{j} \in\right] 0, f_{j}\left[\right.$ and $\left.\widetilde{b}_{j}^{ \pm} \in\right] 0, \varepsilon \min \left\{f_{j}-\widetilde{a}_{j}, g_{j}^{ \pm}\right\}[$, $j=1, \ldots, n$.

Proof. The result follows by Corollary 2.8 and the fact that, in the definition of $\mathrm{OP}(p) u$, each $c_{j}<f_{j}$ can be chosen arbitrarily close to $f_{j}$.

Remark 2.10. If $m_{j}$ is real and $p_{j} \in S_{\mathrm{r}-\mathrm{a}}^{m_{j}, \varepsilon}$ for $j=1,2$, then the symbol of the compound $\operatorname{OPS}\left(p_{1}\right) \operatorname{OPS}\left(p_{2}\right)$ is in $S_{\mathrm{r}-\mathrm{a}}^{m_{1}+m_{2}, \varepsilon}$. Indeed, the symbols $p_{j}$ only depend on the cotangent variable $\xi \in \mathbb{R}^{n}$, so the symbol of the compound $\operatorname{OPS}\left(p_{1}\right) \operatorname{OPS}\left(p_{2}\right)$ is the function $p_{1} p_{2}$. By the standard calculus of pseudodifferential operators, $p_{1} p_{2} \in$ $S^{m_{1}+m_{2}}$. The function $p_{1} p_{2}$ is analytic on $K_{\varepsilon}^{n}$, and finally we have $\left|\left(p_{1} p_{2}\right)(\zeta)\right| \leq$ $\left\|p_{1}\right\|_{*}^{\left(m_{1}\right)}\left\|p_{2}\right\|_{*}^{\left(m_{2}\right)}(1+|\operatorname{Re} \zeta|)^{m_{1}+m_{2}}$ for $\zeta \in K_{\varepsilon}^{n}$.

\section{Proof of Theorem 1.1}

To prove Theorem 1.1, we first relate the Cauchy data

$$
U_{0}=\lim _{x_{n+1} \searrow 0} u \quad \text { and } \quad U_{1}=\lim _{x_{n+1} \searrow 0} \partial_{n+1} u
$$

in terms of the action of the components of the associated Calderón projector. This part of the analysis results in the set of equations (3.3), and it is an adaptation of the development in Hörmander [6, pp. 234-236]. Theorem 2.9 is then used to estimate the domain of analytic continuation of some of the terms in (3.3). We use the notation of Section 1 Also, $\delta$ is the Dirac measure and $\delta^{\prime}$ is its derivative. Finally, $\gamma_{0}^{+} v$ is the trace $\lim _{x_{n+1} \searrow 0} v$.

Let $u^{\circ}$ be the extension by zero of $u$ from $\overline{\tau(a)} \times[0, \lambda]$ to $\mathbb{R}^{n+1}$, and write $U_{0}=u^{\circ}(\cdot, 0)$ and $U_{1}=\left(\partial_{n+1} u^{\circ}\right)(\cdot, 0)$. Fix $R>2 k /\left(1-\varepsilon^{2}\right)$, and let $\chi$ be an element of $C^{\infty}\left(\mathbb{R}^{n+1}\right)$ satisfying

$$
\chi(\xi)=\left\{\begin{array}{ll}
0, & |\xi| \leq \sqrt{1-\varepsilon^{2}} R / 2, \\
1, & |\xi| \geq \sqrt{1-\varepsilon^{2}} R,
\end{array} \quad \xi \in \mathbb{R}^{n+1} .\right.
$$

The function $\chi-1$ is an element of the space $S^{-\infty}\left(\mathbb{R}^{n+1}\right)$ of rapidly decreasing symbols. We readily find by iteration that, for each $\alpha \in \mathbb{N}_{0}^{n+1}, \partial^{\alpha}\left(k^{2}-|\xi|^{2}\right)^{-1}=$ $O\left(|\xi|^{-2-|\alpha|}\right)$ as $|\xi| \rightarrow \infty$, so the function $q$, defined by

$$
q(\xi)=\frac{\chi(\xi)}{k^{2}-|\xi|^{2}}, \quad \xi \in \mathbb{R}^{n+1},
$$


is an element of the space $S^{-2}\left(\mathbb{R}^{n+1}\right)$. The corresponding pseudodifferential operator $Q=\operatorname{OPS}(q)$ is a left parametrix of the Helmholtz operator on $\mathbb{R}^{n+1}$, in that the symbol of the compound $Q\left(\Delta+k^{2}\right)$ is the function $\chi$ (the symbol of the compound actually is $\chi$ and is not merely asymptotically equivalent to $\chi$, since the symbol $k^{2}-|\xi|^{2}$ of the Helmholtz operator depends only on the cotangent variables $\xi_{1}, \ldots, \xi_{n+1}$.) In the sense of distributions in $\mathscr{D}^{\prime}\left(\mathbb{R}^{n+1}\right)$, we have

$$
\begin{aligned}
\left(\Delta+k^{2}\right) u^{\circ} & =\sum_{j=1}^{n}\left(\left.u^{\circ}\right|_{x_{j}=-a_{j}} \otimes \delta^{\prime}\left(x_{j}+a_{j}\right)+\left.u^{\circ}\right|_{x_{j}=a_{j}} \otimes \delta^{\prime}\left(x_{j}-a_{j}\right)\right) \\
& +\sum_{j=1}^{n}\left(\left.\left(\partial_{j} u^{\circ}\right)\right|_{x_{j}=-a_{j}} \otimes \delta\left(x_{j}+a_{j}\right)+\left.\left(\partial_{j} u^{\circ}\right)\right|_{x_{j}=a_{j}} \otimes \delta\left(x_{j}-a_{j}\right)\right) \\
& +U_{0} \otimes \delta^{\prime}\left(x_{n+1}\right)+\left.u^{\circ}\right|_{x_{n+1}=\lambda} \otimes \delta^{\prime}\left(x_{n+1}-\lambda\right) \\
& +U_{1} \otimes \delta\left(x_{n+1}\right)+\left.\left(\partial_{n+1} u^{\circ}\right)\right|_{x_{n+1}=\lambda} \otimes \delta\left(x_{n+1}-\lambda\right) .
\end{aligned}
$$

Applying the compound $\gamma_{0}^{+} Q$ to both sides of (3.1) gives

$$
\left(I-\Pi_{00}^{+}\right) U_{0}+\gamma_{0}^{+} \operatorname{OPS}(\chi-1) u^{\circ}=\Pi_{01}^{+} U_{1}+S_{1}+S_{2},
$$

where the operators

$$
\Pi_{00}^{+}=\gamma_{0}^{+} Q\left((\cdot) \otimes \delta^{\prime}\left(x_{n+1}\right)\right) \quad \text { and } \quad \Pi_{01}^{+}=\gamma_{0}^{+} Q\left((\cdot) \otimes \delta\left(x_{n+1}\right)\right)
$$

are components of the Calderón projector associated with the setup of Theorem 1.1. and where

$$
S_{1}=\sum_{j=1}^{n}\left[\gamma_{0}^{+} Q\left(\left.u^{\circ}\right|_{x_{j}=-a_{j}} \otimes \delta^{\prime}\left(x_{j}+a_{j}\right)\right)+\gamma_{0}^{+} Q\left(\left.\left(\partial_{j} u^{\circ}\right)\right|_{x_{j}=-a_{j}} \otimes \delta\left(x_{j}+a_{j}\right)\right)\right]
$$

as well as

$$
\begin{aligned}
S_{2}= & \sum_{j=1}^{n}\left[\gamma_{0}^{+} Q\left(\left.u^{\circ}\right|_{x_{j}=a_{j}} \otimes \delta^{\prime}\left(x_{j}-a_{j}\right)\right)+\gamma_{0}^{+} Q\left(\left.\left(\partial_{j} u^{\circ}\right)\right|_{x_{j}=a_{j}} \otimes \delta\left(x_{j}-a_{j}\right)\right)\right] \\
& +\gamma_{0}^{+} Q\left(\left.u^{\circ}\right|_{x_{n+1}=\lambda} \otimes \delta^{\prime}\left(x_{n+1}-\lambda\right)\right)+\gamma_{0}^{+} Q\left(\left.\left(\partial_{j} u^{\circ}\right)\right|_{x_{n+1}=\lambda} \otimes \delta\left(x_{n+1}-\lambda\right)\right) .
\end{aligned}
$$

If $I-\Pi_{00}^{+}$and $\Pi_{01}^{+}$have inverses, then (3.2) implies

$$
\left\{\begin{aligned}
U_{0} & =\left(I-\Pi_{00}^{+}\right)^{-1} \Pi_{01}^{+} U_{1} \\
& +\left(I-\Pi_{00}^{+}\right)^{-1}\left(S_{1}+S_{2}\right)+\left(I-\Pi_{00}^{+}\right)^{-1} \gamma_{0}^{+} \operatorname{OPS}(1-\chi) u^{\circ} \\
U_{1} & =\left(\Pi_{01}^{+}\right)^{-1}\left(I-\Pi_{00}^{+}\right) U_{0} \\
& -\left(\Pi_{01}^{+}\right)^{-1}\left(S_{1}+S_{2}\right)+\left(\Pi_{01}^{+}\right)^{-1} \gamma_{0}^{+} \operatorname{OPS}(\chi-1) u^{\circ}
\end{aligned}\right.
$$

We shall use (3.3) to relate the domains of analytic continuability of the Dirichlet datum $U_{0}$ and the Neumann datum $U_{1}$. To this end, we first show that the inverses $\left(I-\Pi_{00}^{+}\right)^{-1}$ and $\left(\Pi_{01}^{+}\right)^{-1}$ exist and have suitable real-analytic symbols. Fix $j \in$ $\{1, \ldots, n+1\}, l \in\{0,1\}$ and $C \in \mathbb{R}$.

Lemma 3.1. For each fixed $x_{j} \in \mathbb{R}$, the operator $Q\left((\cdot) \otimes \delta^{(l)}\left(x_{j}-C\right)\right)$ has a symbol $\pi_{l, j}\left(\cdot, x_{j}-C\right) \in S_{\mathrm{r}-\mathrm{a}}^{l-1, \varepsilon}$. The function $\pi_{l, j}\left(\xi_{(j)}, s\right)$ is analytically continuable in $s$ to the domain $\{s \in \mathbb{C}$, $\operatorname{Re} s \neq 0\}$. 
Proof. Following Hörmander [6, p. 109], we will consider a function $\phi \in C_{0}^{\infty}(]-1,1[)$ satisfying $\int_{t \in \mathbb{R}} \phi(t)=1$. If $V \in C_{0}^{\infty}\left(\mathbb{R}^{n}\right)$, then

$$
\begin{aligned}
Q\left(V \otimes \delta^{(l)}\left(x_{j}-C\right)\right)(x) & =\lim _{\kappa \rightarrow 0} Q\left(V \kappa^{-1} \phi^{(l)}\left(\left(x_{j}-C\right) / \kappa\right)\right)(x) \\
= & (2 \pi)^{-n} \int_{\xi_{(j)} \in \mathbb{R}^{n}} e^{i \xi_{(j)} x_{(j)}} \widehat{V}\left(\xi_{(j)}\right) \pi_{l, j}\left(\xi_{(j)}, x_{j}-C\right),
\end{aligned}
$$

with the one-parameter family of symbols $\pi_{l, j}(\cdot, s)$ given by

$$
\pi_{l, j}\left(\xi_{(j)}, s\right)=\frac{1}{2 \pi} \lim _{\kappa \rightarrow 0} \int_{t \in \mathbb{R}}(i t)^{l} q\left(\xi_{(j)}, t\right) e^{i t s} \widehat{\phi}(\kappa t), \quad \xi_{(j)} \in \mathbb{R}^{n}, s \in \mathbb{R} .
$$

Writing $\rho=\sqrt{1-\varepsilon^{2}} R$ and $\widetilde{q}\left(\xi_{(j)}, t\right)=\left(k^{2}-\left|\xi_{(j)}\right|^{2}-t^{2}\right)^{-1}$, we get, for $s \neq 0$,

$$
\int_{t \in \mathbb{R}} t^{l} q\left(\xi_{(j)}, t\right) e^{i t s} \widehat{\phi}(\kappa t)=\int_{|t|>\rho} \widetilde{q}\left(\xi_{(j)}, t\right) t^{l} e^{i t s} \widehat{\phi}(\kappa t)+\int_{|t| \leq \rho} t^{l} q\left(\xi_{(j)}, t\right) e^{i t s} \widehat{\phi}(\kappa t) .
$$

(Recall that $\chi\left(\xi_{(j)}, t\right)=1$ and thus $q\left(\xi_{(j)}, t\right)=\widetilde{q}\left(\xi_{(j)}, t\right)$ when $\left|\xi_{(j)}\right|^{2}+t^{2}>\rho^{2}$.) The function $\mathbb{C} \ni t \mapsto t^{l} \widetilde{q}\left(\xi_{(j)}, t\right) e^{i t\left(x_{j}-d\right)} \widehat{\phi}(\kappa t)$ is analytic in $\{t \in \mathbb{C},|t|>\rho\}$ and exponentially decreasing w.r.t. $|t|$ when $\operatorname{sgn} \operatorname{Im} t=\operatorname{sgn} s$, so

$$
\int_{|t|>\rho} \widetilde{q}\left(\xi_{(j)}, t\right) t^{l} e^{i t s} \widehat{\phi}(\kappa t)=-\int_{\phi=\pi \operatorname{sgn} s}^{0} \widetilde{q}\left(\xi_{(j)}, \rho e^{i \phi}\right) i\left(\rho e^{i \phi}\right)^{l+1} e^{i \rho \exp (i \phi) s} \widehat{\phi}\left(\kappa \rho e^{i \phi}\right),
$$

and consequently

$$
2 \pi i^{-l} \pi_{l, j}\left(\xi_{(j)}, s\right)=\int_{|t| \leq \rho} t^{l} q\left(\xi_{(j)}, t\right) e^{i t s}+\int_{\phi=0}^{\pi \operatorname{sgn} s} \widetilde{q}\left(\xi_{(j)}, \rho e^{i \phi}\right) i\left(\rho e^{i \phi}\right)^{l+1} e^{i \rho \exp (i \phi) s} .
$$

In particular, the mapping $\mathbb{R} \backslash\{0\} \ni s \mapsto \pi_{l, j}\left(\xi_{(j)}, s\right)$ is analytically continuable to the complex plane without the imaginary axis. Also, for $\left|\xi_{(j)}\right|>\rho$ and $s \in \mathbb{R} \backslash\{0\}$ we have

$$
\begin{aligned}
2 \pi i^{-l} \pi_{l, j}\left(\xi_{(j)}, s\right)= & 2 \pi i(\operatorname{sgn} s) \sum_{t}^{\operatorname{sgn} s} \operatorname{Res} \widetilde{q}\left(\xi_{(j)}, t\right) t^{l} e^{i t s} \\
& =-\frac{\pi\left((\operatorname{sgn} s) i \sqrt{\left|\xi_{(j)}\right|^{2}-k^{2}}\right)^{l} e^{-|s| \sqrt{\left|\xi_{(j)}\right|^{2}-k^{2}}}}{\sqrt{\left|\xi_{(j)}\right|^{2}-k^{2}}},
\end{aligned}
$$

where $\sum_{t}^{+}$Res and $\sum_{t}^{-}$Res signify the sum of residues w.r.t. $t$ in the upper and in the lower complex half-plane, respectively. Thus, for nonzero real $s$, the symbol $\pi_{l, j}(\cdot, s)$ is in $S^{-\infty}$. There is a jump discontinuity in (3.4) across $s=0$, but we readily find that both $\lim _{s \nearrow_{0}} \pi_{l, j}(\cdot, s)$ and $\lim _{s \searrow_{0}} \pi_{l, j}(\cdot, s)$ are in $S^{l-1}$. Also, if the real part of a component of $\zeta_{(j)} \in K_{\varepsilon}^{n}$ is greater than $R$, then $\left|\operatorname{Re} \zeta_{(j)}\right|>R$ and

$$
\operatorname{Re}\left[\left(\zeta_{(j)}\right)^{2}\right]-k^{2}>\left(1-\varepsilon^{2}\right)\left|\operatorname{Re} \zeta_{(j)}\right|^{2}-k^{2}>\left(1-\varepsilon^{2}\right) R^{2}-k^{2}>0,
$$

so the right-hand side in (3.4) is analytic on $K_{\varepsilon}^{n}$. Finally, for such $\zeta_{(j)}$, we have

$$
\begin{aligned}
\left|\left[\left(\zeta_{(j)}\right)^{2}-k^{2}\right]^{1 / 2}\right| & =\sqrt{\left.|| \operatorname{Re} \zeta_{(j)}\right|^{2}-\left|\operatorname{Im} \zeta_{(j)}\right|^{2}-k^{2}+2 i \operatorname{Re} \zeta_{(j)} \cdot \operatorname{Im} \zeta_{(j)} \mid} \\
& \geq \sqrt{\left|\operatorname{Re} \zeta_{(j)}\right|^{2}\left(1-\varepsilon^{2}\right)-k^{2}} \geq C\left(1+\left|\operatorname{Re} \zeta_{(j)}\right|\right)
\end{aligned}
$$

when

$$
C \leq \frac{k \sqrt{3\left(1-\varepsilon^{2}\right)}}{2 k+\sqrt{1-\varepsilon^{2}}}
$$

so $\pi_{l, j}(\cdot, s) \in S_{\mathrm{r}-\mathrm{a}}^{l-1, \varepsilon}$ for every real $s$. 
Corollary 3.2. The symbol of the operator $\gamma_{0}^{+} Q\left((\cdot) \otimes \delta^{(l)}\left(x_{n+1}-C\right)\right)$ is the function $\lim _{x_{n+1} \searrow 0} \pi_{l, n+1}\left(\cdot, x_{n+1}-C\right) \in S_{\mathrm{r}-\mathrm{a}}^{l-1, \varepsilon}$. In particular, $I-\Pi_{00}^{+}$and $\Pi_{01}^{+}$have symbols $1-\lim _{x_{n+1} \searrow 0} \pi_{1, n+1}\left(\cdot, x_{n+1}\right) \in S_{\mathrm{r}-\mathrm{a}}^{0, \varepsilon} \quad$ and $\quad \lim _{x_{n+1} \searrow 0} \pi_{0, n+1}\left(\cdot, x_{n+1}\right) \in S_{\mathrm{r}-\mathrm{a}}^{-1, \varepsilon}$, respectively.

Lemma 3.3. $I-\Pi_{00}^{+}$and $\Pi_{01}^{+}$have inverses with symbols in $S_{\mathrm{r}-\mathrm{a}}^{0, \varepsilon}$ and $S_{\mathrm{r}-\mathrm{a}}^{1, \varepsilon}$, respectively.

Proof. For $\xi^{\prime} \in \mathbb{R}^{n}$ with $\left|\xi^{\prime}\right|>\sqrt{1-\varepsilon^{2}} R$, we have

$1-\lim _{x_{n+1} \searrow 0} \pi_{1, n+1}\left(\xi^{\prime}, x_{n+1}\right)=\frac{1}{2} \quad$ and $\quad \lim _{x_{n+1} \searrow 0} \pi_{0, n+1}\left(\xi^{\prime}, x_{n+1}\right)=-\frac{1}{2 \sqrt{\left|\xi^{\prime}\right|^{2}-k^{2}}}$.

Thus, the symbols $\mathbb{R}^{n} \ni \xi^{\prime} \mapsto 2 \chi\left(\xi^{\prime}, 0\right)$ and $\mathbb{R}^{n} \ni \xi^{\prime} \mapsto-2 \sqrt{\left|\xi^{\prime}\right|^{2}-k^{2}} \chi\left(\xi^{\prime}, 0\right)$ define inverses $\left(I-\Pi_{00}^{+}\right)^{-1}$ and $\left(\Pi_{01}^{+}\right)^{-1}$, respectively. Trivially, the symbol of $\left(I-\Pi_{00}^{+}\right)^{-1}$ is in $\mathrm{S}_{\mathrm{r}-\mathrm{a}}^{0, \varepsilon}$. Also, if the real part of a component of $\zeta^{\prime} \in K_{\varepsilon}^{n}$ is greater than $R$, then

$$
\begin{aligned}
\left|\left(\left(\zeta^{\prime}\right)^{2}-k^{2}\right)^{1 / 2}\right| & =\sqrt{\left.|| \operatorname{Re} \zeta^{\prime}\right|^{2}-\left|\operatorname{Im} \zeta^{\prime}\right|^{2}-k^{2}+2 i \operatorname{Re} \zeta^{\prime} \cdot \operatorname{Im} \zeta^{\prime} \mid} \\
& \leq \sqrt{\left.|| \operatorname{Re} \zeta^{\prime}\right|^{2}+2 i \operatorname{Re} \zeta^{\prime} \cdot \operatorname{Im} \zeta^{\prime} \mid} \leq \sqrt{|1+2 i \varepsilon|}|\operatorname{Re} \zeta|<5^{1 / 4}(1+|\operatorname{Re} \zeta|),
\end{aligned}
$$

and in conclusion the symbol of $\left(\Pi_{01}^{+}\right)^{-1}$ is in $\mathrm{S}_{\mathrm{r}-\mathrm{a}}^{1, \varepsilon}$.

We next estimate the analytic continuability of terms of the forms

$$
\left(I-\Pi_{00}^{+}\right)^{-1} \gamma_{0}^{+} Q\left(\left.u^{\circ}\right|_{x_{j}=C} \otimes \delta^{(l)}\left(x_{j}-C\right)\right), \quad\left(\Pi_{01}^{+}\right)^{-1} \gamma_{0}^{+} Q\left(\left.u^{\circ}\right|_{x_{j}=C} \otimes \delta^{(l)}\left(x_{j}-C\right)\right)
$$

occurring in (3.3). First assume $j=n+1$. Remark 2.10, Corollary 3.2 and Lemma 3.3 imply that the compounds $\left(I-\Pi_{00}^{+}\right)^{-1} \gamma_{0}^{+} Q\left((\cdot) \otimes \delta^{(l)}\left(x_{j}-C\right)\right)$ and $\left(\Pi_{01}^{+}\right)^{-1} \gamma_{0}^{+} Q\left((\cdot) \otimes \delta^{(l)}\left(x_{j}-C\right)\right)$ have symbols in $S_{\mathrm{r}-\mathrm{a}}^{l-\varepsilon}$ and $S_{\mathrm{r}-\mathrm{a}}^{l, \varepsilon}$, respectively. Thus, by Theorem 2.9 the functions in (3.5) are analytically continuable to each complex polyrectangle $T\left(\widetilde{a}, \widetilde{b}^{ \pm}\right)$with $\left.\widetilde{a}_{j} \in\right] 0, a_{j}\left[\right.$ and $\left.\widetilde{b}_{j}^{ \pm} \in\right] 0, \varepsilon \min \left\{a_{j}-\widetilde{a}_{j}, b_{j}^{ \pm}\right\}[, j=1, \ldots, n$.

Now assume $j \neq n+1$ and let $d$ be the symbol of $\left(I-\Pi_{00}^{+}\right)^{-1}$ or of $\left(\Pi_{01}^{+}\right)^{-1}$. Abbreviate $\xi^{\prime}=\xi_{(n+1)}, \xi^{\prime \prime}=\xi_{(j)}, \xi^{\prime \prime \prime}=\xi_{(j, n+1)}$, and similarly for other vectors. For $x^{\prime}=x_{(n+1)} \in \mathbb{R}^{n}$ we have

$$
\begin{aligned}
& (2 \pi)^{2 n} \operatorname{OPS}(d) \gamma_{0}^{+} Q\left(\left.u^{\circ}\right|_{x_{j}=C} \otimes \delta^{(l)}\left(x_{j}-C\right)\right)\left(x^{\prime}\right) \\
& =\left.\iiint \int_{w^{\prime}, \eta^{\prime}, y^{\prime \prime}, \xi^{\prime \prime} \in \mathbb{R}^{n}} e^{i \eta^{\prime}\left(x^{\prime}-w^{\prime}\right)+i \xi^{\prime \prime \prime} w^{\prime \prime \prime}-i \xi^{\prime \prime} y^{\prime \prime}} d\left(\eta^{\prime}\right) \pi_{l, j}\left(\xi^{\prime \prime}, x_{j}-C\right) u^{\circ}\right|_{y_{j}=C}\left(y^{\prime \prime}\right) \\
& =\left.2 \pi \iiint \int_{\eta^{\prime}, y^{\prime \prime}, \xi^{\prime \prime} \in \mathbb{R}^{n}, w_{j} \in \mathbb{R}} e^{i \eta_{j}\left(x_{j}-w_{j}\right)+i \eta^{\prime \prime \prime} x^{\prime \prime \prime}-i \xi^{\prime \prime} y^{\prime \prime}} d\left(\eta^{\prime}\right) \pi_{l, j}\left(\xi^{\prime \prime}, x_{j}-C\right) u^{\circ}\right|_{y_{j}=C}\left(y^{\prime \prime}\right) \delta\left(\xi^{\prime \prime \prime}-\eta^{\prime \prime \prime}\right) \\
& =\left.(2 \pi)^{2} \iiint_{y^{\prime \prime}, \xi^{\prime \prime} \in \mathbb{R}^{n}, \eta_{j} \in \mathbb{R}} e^{i \eta_{j} x_{j}+i \xi^{\prime \prime \prime} x^{\prime \prime \prime}-i \xi^{\prime \prime} y^{\prime \prime}} d\left(\xi^{\prime \prime \prime}, \eta_{j}\right) \pi_{l, j}\left(\xi^{\prime \prime}, x_{j}-C\right) u^{\circ}\right|_{y_{j}=C}\left(y^{\prime \prime}\right) \delta\left(\eta_{j}\right) \\
& =(2 \pi)^{n+2}\left[\left.\gamma_{0}^{+} \operatorname{OPS}\left(d\left(\xi^{\prime \prime \prime}, 0\right) \pi_{l, j}\left(\xi^{\prime \prime}, x_{j}-C\right)\right) u^{\circ}\right|_{x_{j}=C}\right]\left(x^{\prime}\right) .
\end{aligned}
$$

By Remark 2.10, Lemma 3.1 and Lemma 3.3, for each fixed $x_{j}$ the symbol $\mathbb{R}^{n} \ni$ $\xi^{\prime \prime} \mapsto d\left(\xi^{\prime \prime \prime}, 0\right) \pi_{l, j}\left(\xi^{\prime \prime}, x_{j}-C\right)$ is in $S_{\mathrm{r}-\mathrm{a}}^{l, \varepsilon}$, and it is analytically continuable to $\left\{x_{j} \in \mathbb{C}, \operatorname{Re} x_{j} \neq C\right\}$. Finally, using Theorem 2.9. we obtain at least the same estimates on the analytic continuability of the terms (3.5) as in the case $j=n+1$. 
It remains to estimate the analytic continuability of the terms

$$
\left(I-\Pi_{00}^{+}\right)^{-1} \gamma_{0}^{+} \operatorname{OPS}(1-\chi) u^{\circ} \text { and }\left(\Pi_{01}^{+}\right)^{-1} \gamma_{0}^{+} \operatorname{OPS}(\chi-1) u^{\circ}
$$

occurring in (3.3). Again write $d$ for the symbol of $\left(I-\Pi_{00}^{+}\right)^{-1}$ or of $\left(\Pi_{01}^{+}\right)^{-1}$. We readily find that $\operatorname{OPS}(d) \gamma_{0}^{+} \operatorname{OPS}(\chi-1) u^{\circ}=\gamma_{0}^{+} \operatorname{OPS}\left(d\left(\xi^{\prime}\right)(\chi(\xi)-1)\right) u^{\circ}$. The symbol $d\left(\xi^{\prime}\right)(\chi(\xi)-1)$ is in $C_{0}^{\infty}\left(\mathbb{R}^{n+1}\right)$, and the function $u^{\circ}$ is bounded and compactly supported in $\mathbb{R}^{n+1}$. The Fourier transform $\widehat{u}^{\circ}$ of $u^{\circ}$ is in $C^{\infty}\left(\mathbb{R}^{n+1}\right)$, so the function $d \cdot(\chi-1) \cdot \widehat{u}^{\circ}$ is smooth and compactly supported. The Fourier-Laplace transform $v$ of $d \cdot(\chi-1) \cdot \widehat{u}^{\circ}$ is then an entire function on $\mathbb{C}^{n+1}$. But for every $x$ in $\mathbb{R}^{n+1}$,

$$
(2 \pi)^{n+1} \operatorname{OPS}\left(d\left(\xi^{\prime}\right)(\chi(\xi)-1)\right) u^{\circ}(x)=\int_{\mathbb{R}^{n+1}} e^{i x \xi} d\left(\xi^{\prime}\right)(\chi(\xi)-1) \widehat{u}^{\circ}(\xi) d \xi=v(-x),
$$

so the function $\operatorname{OPS}\left(d\left(\xi^{\prime}\right)(\chi(\xi)-1)\right) u^{\circ}$ is analytically continuable to the whole complex space $\mathbb{C}^{n+1}$. In particular, its restriction $\gamma_{0}^{+} \operatorname{OPS}\left(d\left(\xi^{\prime}\right)(\chi(\xi)-1)\right) u^{\circ}$ is analytically continuable to the 'polystrip' $T\left(a^{\prime}, \pm \infty\right)$.

Theorem 1.1 now follows from (3.3) and the fact that the constant $\varepsilon$ can be chosen arbitrarily close to 1 in the analysis in this section.

\section{Outlook}

We expect that the method of proof of Theorem 1.1 presented here can be extended to handle general linear, second-order, analytic, elliptic differential operators in two or more independent variables, as well as general analytic pieces of the boundary. This requires an analysis similar to that of Section 2 but done on a subset of standard pseudodifferential operators with symbols that are real-analytic and dependent on the full variable set $(x, \xi)$.

\section{REFERENCES}

[1] Louis Boutet de Monvel, Opérateurs pseudo-différentiels analytiques et opérateurs d'ordre infini (French), Ann. Inst. Fourier (Grenoble) 22 (1972), no. 3, 229-268. MR.0341189 (49 \#5939)

[2] A. Doicu, Yu. Eremin, and T. Wriedt, Acoustic \& Electromagnetic Scattering Analysis Using Discrete Sources, Academic Press, 2000.

[3] Graeme Fairweather and Andreas Karageorghis, The method of fundamental solutions for elliptic boundary value problems, Numerical treatment of boundary integral equations, Adv. Comput. Math. 9 (1998), no. 1-2, 69-95, DOI 10.1023/A:1018981221740. MR.1662760 (2000a:65165)

[4] Gerald B. Folland, Real analysis, Modern techniques and their applications; A WileyInterscience Publication. Pure and Applied Mathematics (New York), John Wiley \& Sons, Inc., New York, 1984. MR767633 (86k:28001)

[5] Lars Hörmander, The analysis of linear partial differential operators. I, Distribution theory and Fourier analysis, Grundlehren der Mathematischen Wissenschaften [Fundamental Principles of Mathematical Sciences], vol. 256, Springer-Verlag, Berlin, 1983. MR717035 (85g:35002a)

[6] Lars Hörmander, The analysis of linear partial differential operators. III, Pseudodifferential operators, Grundlehren der Mathematischen Wissenschaften [Fundamental Principles of Mathematical Sciences], vol. 274, Springer-Verlag, Berlin, 1985. MR781536 (87d:35002a)

[7] R. Kangro and U. Kangro, Extendability of solutions of Helmholtz's equation across a hyperplane in three dimensions, Proc. of the International Conf. on Computational and Mathematics Methods in Science and Engineering, CMMSE 2006 (Madrid), 21-25 September 2006, pp. 433-437.

[8] M. Karamehmedović, P.-E. Hansen, K. Dirscherl, E. Karamehmedović, and T. Wriedt, Profile estimation for Pt submicron wire on rough Si substrate from experimental data, Opt. Express 20 (2012), 21678-21686. 
[9] M. Karamehmedović, P.-E. Hansen, and T. Wriedt, An efficient scattering model for PEC and penetrable nanowires on a dielectric substrate, J. Eur. Opt. Soc. Rapid 6 (2011), 11021.

[10] _ A fast inversion method for highly conductive submicron wires on a substrate, J. Eur. Opt. Soc. Rapid 6 (2011), 11039.

[11] M. Karamehmedović, M.-P. Sørensen, P.-E. Hansen, and A. V. Lavrinenko, Application of the method of auxiliary sources to a defect-detection inverse problem of optical diffraction microscopy, J. Eur. Opt. Soc. Rapid 5 (2010), 10021.

[12] Gottfried Köthe, Topological vector spaces. II, Grundlehren der Mathematischen Wissenschaften [Fundamental Principles of Mathematical Science], vol. 237, Springer-Verlag, New York-Berlin, 1979. MR551623 (81g:46001)

[13] John M. Lee, Introduction to smooth manifolds, Graduate Texts in Mathematics, vol. 218, Springer-Verlag, New York, 2003. MR1930091 (2003k:58001)

[14] R. F. Millar, The location of singularities of two-dimensional harmonic functions. I. Theory, SIAM J. Math. Anal. 1 (1970), 333-344. MR0275759 (43 \#1512)

[15] R. F. Millar, The location of singularities of two-dimensional harmonic functions. II. Applications, SIAM J. Math. Anal. 1 (1970), 345-353. MR0275760 (43 \#1513)

[16] R. F. Millar, Singularities of solutions to linear, second order analytic elliptic equations in two independent variables. I. The completely regular boundary, Applicable Anal. 1 (1971), no. 2, 101-121. MR0287132 (44 \#4339)

[17] R. F. Millar, Singularities of two-dimensional exterior solutions of the Helmholtz equation, Proc. Cambridge Philos. Soc. 69 (1971), 175-188. MR0268534 (42 \#3431)

[18] R. F. Millar, Singularities of solutions to linear, second order, analytic elliptic equations in two independent variables. II. The piecewise regular boundary, Applicable Anal. 2 (1972/73), 301-320. MR0390483 (52 \#11309)

[19] R. F. Millar, Singularities of solutions to exterior analytic boundary value problems for the Helmholz equation in three independent variables. I. The plane boundary, SIAM J. Math. Anal. 7 (1976), no. 1, 131-156. MR0390462 (52 \#11288)

[20] R. F. Millar, Singularities of solutions to exterior analytic boundary value problems for the Helmholtz equation in three independent variables. II. The axisymmetric boundary, SIAM J. Math. Anal. 10 (1979), no. 4, 682-694, DOI 10.1137/0510063. MR533939 (81m:35010)

[21] Robert F. Millar, The analytic continuation of solutions to elliptic boundary value problems in two independent variables, J. Math. Anal. Appl. 76 (1980), no. 2, 498-515, DOI 10.1016/0022247X(80)90045-1. MR587358 (81j:35025)

[22] Robert F. Millar, Singularities and the Rayleigh hypothesis for solutions to the Helmholtz equation, IMA J. Appl. Math. 37 (1986), no. 2, 155-171, DOI 10.1093/imamat/37.2.155. MR $983524(90 \mathrm{~b}: 35187)$

[23] Raul Kangro, Urve Kangro, and Roy Nicolaides, Extendability of solutions of Helmholtz's equation to the interior of a two-dimensional scatterer, Quart. Appl. Math. 58 (2000), no. 3, 591-600. MR 1770657 (2001f:35094)

[24] Boris Sternin and Victor Shatalov, Differential equations on complex manifolds, Mathematics and its Applications, vol. 276, Kluwer Academic Publishers Group, Dordrecht, 1994. MR $1391964(97 \mathrm{~d}: 58192)$

[25] B. Yu. Sternin and V. E. Shatalov, Analytic continuation of solutions of integral equations and localization of singularities (Russian, with Russian summary), Differ. Uravn. 32 (1996), no. 11, 1544-1553; English transl., Differential Equations 32 (1996), no. 11, 1541-1549 (1997). MR 1607109 (99b:35017)

Department of Applied Mathematics and Computer Science, Technical University of Denmark, Matematiktorvet 303B, DK-2800 Kgs. Lyngby, Denmark 